\title{
The Thermohydrodynamic Analysis of Sliding Bearing High-Speed Motorized Spindle by Rotor Dynamic
}

\author{
Songsheng Li , Yuan Shen and Yuxin Lu \\ School of Mechatronic Engineering and Automation, Shanghai Key Laboratory of Intelligent manufacturing and Robotics, Shanghai \\ University, Shanghai University, China
}

\begin{abstract}
This is paper presents thermohydrodynamic characteristics of high speed motorized spindle sliding bearing rotor system. The dynamic characteristic of the oil film bearing is affected by temperature increment, thereby affecting the high-speed spindle rotor system dynamics. This study applied the hydrodynamic lubrication theory, the influence of temperature on the viscosity of lubricating oil, associated with the bearing stiffness, oil film damping and other performance parameters, is considered in generalized Reynolds equation of oil film bearing. The theoretical model of the sliding bearing rotor system is established by using the transfer matrix method to analyze the dynamic characteristic and verified by experiments. The results show the high temperature environment in the motorized spindle and the friction of the bearing lead to oil temperature rise and viscosity reduction, which influences the bearing capacity, stiffness and damping, hence impact on the critical speeds and modal shapes of the sliding bearing rotor system.
\end{abstract}

\section{Introduction}

With the development of science and all kinds of industrial technology, motorized spindle trends to be higher speed and larger power. Hydrodynamic sliding bearing has been widely used for its advantages of large load capacity, long service life, non contact and so on. Therefore, the hydrodynamic sliding bearing motorized spindle came into being. However, the serious friction and large heat generation of the bearing have been the key factors which restrict the development of higher speed [1]. It is a important engineering guarantee to study on the high speed hydrodynamic sliding bearing and analyze the dynamic characteristic of the high-speed motorized spindle sliding bearing rotor system.

Researches on rotating machinery occupies an important position in rotor dynamics, they are the foundation of calculating various rotor dynamics when design and optimize complex rotor system. Traditional rotor dynamics often treat bearings as accessories of the rotor neglecting the bearing itself is a important part in the rotor system [2]-[4]. For the studies of sliding bearing, since $\mathrm{O}$. Reynolds derived the differential equation of oil film pressure distribution in 1886, most researches are based on the isothermal hydrodynamic lubrication model [5]. Whereas, in the actual operation process, because of the lubrication of extrusion and shear viscosity the sliding bearing will friction and generate heat, and the higher the speed, the more heat generated inside the bearing, resulting in high temperature [6], [7]. As the temperature and oil film pressure distribution of the journal bearing shown Fig. 1, the temperature rise leads to the oil viscosity decrease, the viscosity changes will also affect the dynamic pressure of oil film, bearing stiffness and damping etc. [8], [9]. So in the high temperature environment of the high-speed motorized spindle and high speed conditions, the application of traditional isothermal hydrodynamic lubrication model can not accurately reflect the dynamic characteristics of sliding bearing, and can't meet the need of practical engineering [10]-[13].

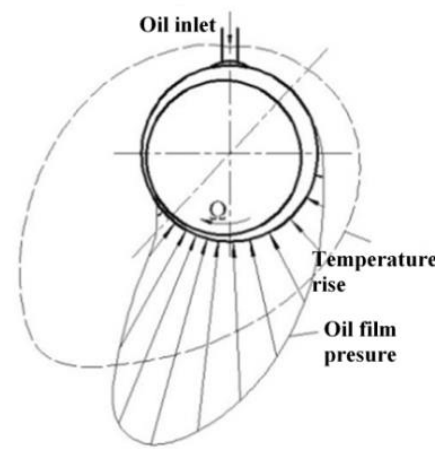

Figure 1. The temperature and oil film pressure distribution of the journal bearing.

A theoretical model, presented in this paper, applies transfer matrix method to analyze the dynamic characteristic of the sliding bearing rotor system. Based on the hydrodynamic lubrication theory, viscositytemperature equation is considered in Reynolds equation to explore the influence of temperature on the viscosity of bearing lubricating oil film. The theoretical analysis 
model of high speed radial dynamic pressure sliding bearing is established combined with the energy equation. The relationship of bearing temperature, rotation speed, stiffness and damping is explored as well as their influence on the critical speed and modal shape. Simplified calculation methods of bearing total stiffness of isotropic and anisotropic sliding bearing rotor system are presented, the critical speed results of the two methods are compared and the first order critical speed is verified via experiment.

\section{Dynamic analysis model of rotor system}

The actual rotor is an elastic system of continuous mass distribution with infinite number of degrees of freedom. In rotor dynamics, the rotor is simplified to a multiple degrees of freedom (MDOF) model with a plurality of lumped mass in order to facilitate the calculation. That is, the mass and the moment of inertia of the rotor are lumped to a number of nodes along the center line of the shaft. These nodes are generally selected in the gear, bearing center, shaft coupling, the section of the shaft diameter changes and the shaft end and so on. The total notes $\mathrm{N}$ have certain requirements, present work shows that to make natural frequency error less than $1 \%$, the number of nodes $\mathrm{N}$ should meet the following formula [14]:

$$
N \geq 1+5.34 v
$$

where, $v$ is the highest order of natural frequencies required to calculate.

\subsection{Isotropic bearing rotor system}

Excluding damping, the bending vibration of the axis deflection curve is a plane curve. The support for the rotor system is isotropic in this case, so only one plane vibration modal is needed to obtain the critical speed and corresponding modal shape. The axial direction is $x$ direction and the radial direction is $z$ direction, and the $x o z$ plane is the vibration plane of rotor.

For the cross-section i of the rotor, the state vector is $Z_{i}$, which is composed of the radial displacement $X_{i}$, the torsion angle $A_{i}$, the bending moment $M_{i}$ and the shear force $Q_{i}$. It can be written as:

$$
Z_{i}=\left[\begin{array}{llll}
X & A & M & Q
\end{array}\right]_{i}^{T}
$$

It has the following relationship with the $i+1$ section the state vector $Z_{i+1}$ of the rotor:

$$
Z_{i+1}=T_{i} Z_{i}
$$

where, $T_{i}$ is the transfer matrix of two sections and is shown as:

$$
T_{i}=\left[\begin{array}{cccc}
1+\frac{l^{3}}{6 E J}(1-v)\left(m \omega^{2}-K_{s j}\right) & l+\frac{l^{2}}{2 E J}\left(I_{p}-I_{d}\right) \omega^{2} & \frac{l^{2}}{2 E J} & \frac{l^{3}}{6 E J}(1-v) \\
\frac{l^{2}}{2 E J}\left(m \omega^{2}-K_{s j}\right) & 1+\frac{l}{E J}\left(I_{p}-I_{d}\right) \omega^{2} & \frac{l}{E J} & \frac{l^{2}}{2 E J} \\
l\left(m \omega^{2}-K_{s j}\right) & \left(I_{p}-I_{d}\right) \omega^{2} & 1 & l \\
\left(m \omega^{2}-K_{s j}\right) & 0 & 0 & 1
\end{array}\right]
$$

where, $E$ is the elastic modulus of materials; $J$ is the section distance; $v$ is coefficient of shear force effect.

It can be seen that the elements of the transfer matrix are the physical parameters of the rotor components and the vortex frequency function. If the section of the rotor is not supported, or regardless the shear effect of the shaft, or regardless of the gyroscopic effect of the disk or swing effect, just make the $K_{s j}$ or $v$ or $I_{p}$ or $I_{d}$ zero respectively.

\subsection{Anisotropy bearing rotor system}

To consider both the motion of the rotor in horizontal and vertical two levels and the damping effect, it needs anisotropy dynamic method. The vibration quantity of the system will occur phase-difference, the magnitude of the vibration amplitude will increase or decrease with time. Therefore, the vibration quantity needs to use the complex number to express, the complex representation of the state vector is [1]:

$$
\mathrm{Z}_{\mathrm{i}}=[f: e]_{i}^{T}=\left[M_{x} Q_{x} M_{y} Q_{y} \vdots X A Y B\right]
$$

Besides the extended to the 8 order square matrix, it is similar to the isotropy.

\section{Thermohydrodynamic lubrication model of bearing}

\subsection{Reynolds equation}

The Reynolds equation of the bearing oil film pressure distribution at steady state is shown as follow on the assumption that the lubricating oil is laminar and incompressible, ignored the weight of the oil layer and the inertia force of the movement.

$$
\frac{\partial}{\partial x}\left(\frac{h^{3}}{\mu} \frac{\partial P}{\partial x}\right)+\frac{\partial}{\partial z}\left(\frac{h^{3}}{\mu} \frac{\partial P}{\partial z}\right)=6 U \frac{\partial h}{\partial x}
$$

where, $x$ is the coordinates along the axis neck circle direction, $z$ is the axial coordinate, $h$ is the radial oil film thickness, $\mu$ is oil dynamic viscosity, $P$ is the oil film pressure, $U$ is the linear velocity of the spindle neck.

\subsection{Energy equation}

Assuming all the generated heat by the bearing is carried away by the lubricating oil, that is apply the adiabatic boundary condition in the bearing and oil film interface. The equation of energy can be expressed as follows [15]:

$$
\begin{aligned}
& \rho\left[\left(\frac{U h}{2}-\frac{h^{3}}{12 \mu} \frac{\partial P}{\partial x}\right) \frac{\partial\left(c_{v} T\right)}{\partial x}-\frac{h^{3}}{12 \mu} \frac{\partial P}{\partial z} \frac{\partial\left(c_{v} T\right)}{\partial z}\right] \\
& =\mu \frac{U^{2}}{h}+\frac{h^{3}}{12 \mu}\left[\left(\frac{\partial P}{\partial x}\right)^{2}+\left(\frac{\partial P}{\partial z}\right)^{2}\right]
\end{aligned}
$$

where, $\rho$ is the oil density, $T$ is the oil temperature, $c_{v}$ is the specific heat capacity of the oil.

Assume the specific heat capacity is constant, the results of the three-dimensional model calculations shows temperature variation is very small along the axial direction and can be neglect [4], that is $\frac{\partial\left(c_{v} T\right)}{\partial z}=0$. So the 
temperature distribution on the surface of the bearing is replaced by the temperature of $\mathrm{z}=0$. By the Reynolds boundary condition, when $\mathrm{z}=0$ the $\frac{\partial T}{\partial z}=0 \frac{\partial P}{\partial z}=0$ and the can be reduced to [16]:

$$
\rho\left(\frac{U h}{2}-\left.\frac{h^{3}}{12 \mu} \frac{\partial P}{\partial x}\right|_{z=0}\right) \frac{d\left(c_{v} T\right)}{d x}=\mu \frac{U^{2}}{h}+\frac{h^{3}}{12 \mu}\left(\left.\frac{\partial P}{\partial x}\right|_{z=0}\right)^{2}
$$

\subsection{The thermohydrodynamic equation}

Lubricating oil viscosity decreased with the increase of temperature quickly. The relationship can be represented by a series of measured value or calculated by empirical formula, commonly used Reynolds temperature viscosity equation is as follows [1]:

$$
\mu=\mu_{0} e^{-\alpha\left(T-T_{0}\right)}
$$

where, $T_{0}$ is the initial temperature of lubricating oil, $\alpha$ is the viscidity temperature index.

The dynamic characteristics of oil film have great influence on the dynamic characteristics of the whole rotor system. Oil film will affect the natural frequency, amplitude and stability of the rotor system. In commonly used calculation, when the oil film force is obtained, the film force is regarded as a function of displacement and velocity near the equilibrium point and expand it use Taylor series. When the axial amplitude is small, the oil film can be simplified to a linear stiffness and damping characteristics. The increment of oil film force can be expressed by the following linear formula [17]:

$$
\left.\begin{array}{l}
\Delta F_{x}=k_{x x} \Delta x+k_{x y} \Delta y+c_{x x} \Delta \dot{x}+c_{x y} \Delta \dot{y} \\
\Delta F_{y}=k_{y x} \Delta x+k_{y y} \Delta y+c_{y x} \Delta \dot{x}+c_{y y} \Delta \dot{y}
\end{array}\right\}
$$

where, $k_{x x}, k_{x y}, k_{y x}, k_{y y}, c_{x x}, c_{x y}, c_{y x}, c_{y y}$ are the 8 oil film dynamic coefficients, the first subscript indicates the direction of the force increment, and the second subscript indicates the direction of displacement or velocity increment.

The three equations using the finite difference method for iterative solution, the oil film pressure distribution of sliding bearing can be obtained by iterative solve the formula (6), (8), (9) using the finite difference method. After obtain the distribution of film pressure, the film stiffness and damping coefficient of the bearing can be obtained by the difference method.

\section{Total stiffness matrix of sliding bearing rotor system}

The bearing rotor is simplified to a certain boundary conditions at the bearing node, where the bearing structure is no longer considered. The oil film stiffness $(\mathrm{K})$ and damping coefficient (C) matrix of sliding bearing as follows:

$$
[K]=\left[\begin{array}{ll}
k_{x x} & k_{x y} \\
k_{y x} & k_{y y}
\end{array}\right] ;[C]=\left[\begin{array}{ll}
c_{x x} & c_{x y} \\
c_{y x} & c_{y y}
\end{array}\right]
$$

\subsection{Total stiffness of isotropy sliding bearing rotor system}

When the stiffness and the equivalent mass of the bearing in the $x$ and $y$ directions are not very different and the coupling is weak, the impact of damping can be neglected and the support is isotropic in the critical speed calculation, namely:

$$
k_{x x}=k_{y y} ; k_{x y}=k_{y x}=0
$$

If the mass and stiffness of the bearing is considered, the oil film and the bearing house are put in series connection. Excluding the coupling of the $x$ and $y$ direction gives [18]:

$$
K_{s j}=\frac{K\left(K_{b}-m_{b} \Omega^{2}\right)}{K+K_{b}-m_{b} \Omega^{2}}
$$

where, $K_{s j}$ is the j-th support of the total stiffness, $K$ is the stiffness of the membrane, $K_{b}$ is the bearing house stiffness, $m_{b}$ is the vibration mass of the bearing house, $\Omega$ is the rotor whirl angular velocity

\subsection{Total stiffness of anisotropy sliding bearing rotor system}

When the stiffness and the equivalent mass of the bearing in the $x$ and $y$ directions are large and the impact of damping can't be neglected, the support is anisotropic. In this case, the total stiffness of $K_{s}$ is in the matrix form, so does the stiffness, damping and mass of the bearing house [18]:

$$
\begin{aligned}
& K_{s}=\left[\begin{array}{ll}
K_{s x x} & K_{s x y} \\
K_{s y x} & K_{s y y}
\end{array}\right]=\left[K_{b}+S C_{b}+S^{2} m_{b}\right] \\
& \cdot\left[K+S C+K_{b}+S C_{b}+S^{2} m_{b}\right]^{-1}[K+S C]
\end{aligned}
$$

where, $S$ is the complex frequency, $S=\lambda+i \Omega$, the real part is the attenuation index and the imaginary part for damping circular frequency, $C_{b}$ is the bearing house damping coefficient matrix.

\section{Analysis results and experimental verification}

\subsection{Results of the thermohydrodynamic model}

The structure of the high-speed sliding bearing motorized spindle is shown in Fig. 2, both the front and the end of the spindle are supported by dynamic pressure sliding bearings. The spindle a simplified at a plurality of lumped mass and the bearing is considered at the model.

According to formula (1), in order to calculate the primary four orders of critical speed, the number of nodes should be divided into at least 23 nodes. In the places where diameter or material changes, or has external load, or supported by bearings and the ends of the spindle should be as nodes. Nodes for this spindle is divided into 24 nodes and is shown in Fig. 3. 


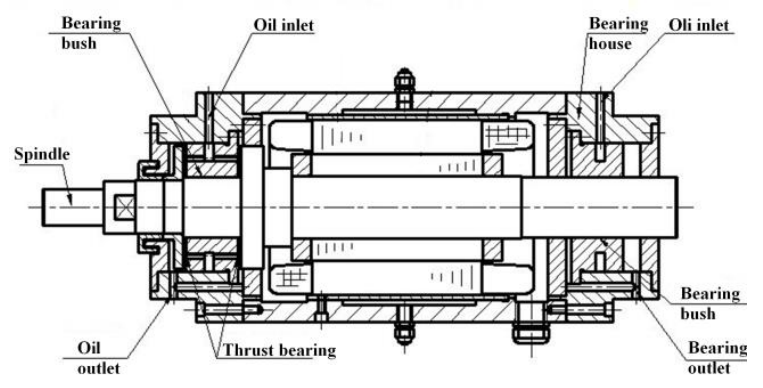

Figure 2. The structure of the sliding bearing motorized spindle.

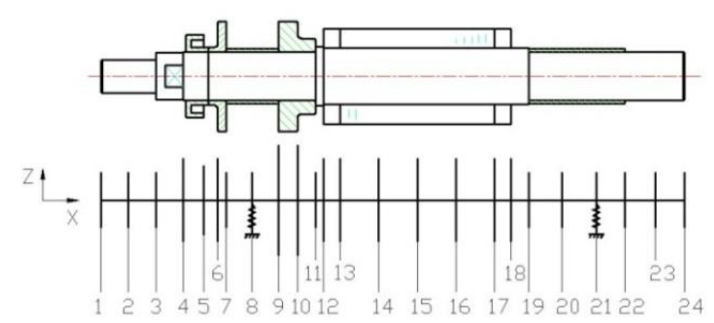

Figure 3. Lumped mass model of the spindle.

It is required to know the sliding bearings characteristics in the critical speed calculation of the sliding rotor system. The sliding bearing structure parameters is shown in Table 1. Fig. 4 shows the steady state temperature of the bearing, whose eccentricity is 0.5 , changes with rotating speed. As shown in the figure, the temperature rises increases significantly when the rotating speed goes up. Since the viscosity of the lubricating oil is closely related to the oil temperature, so the influence of the temperature rise should be considered in the analysis of characteristics of high speed sliding bearing.

Table 1. Structure parameter of the sliding bearing

\begin{tabular}{|lc|c|}
\hline \multicolumn{2}{|c|}{ Parameter } & Value \\
\hline Diameter & $\mathrm{d} / \mathrm{mm}$ & 40 \\
\hline Contact Angle & $\varphi \alpha /{ }^{\circ}$ & 360 \\
\hline Width-diameter ratio & $1 / \mathrm{d}$ & 0.8 \\
\hline Clearance ratio & $\psi$ & 0.002 \\
\hline Average viscosity of lubricating oil & $\mu / \mathrm{Pa} \cdot \mathrm{s}$ & 0.02 \\
\hline Initial viscosity of lubricating oil & $\mu 0 / \mathrm{Pa} \cdot \mathrm{s}$ & 0.038 \\
\hline Initial temperature of lubricating oil & $\mathrm{T} 0 /{ }^{\circ} \mathrm{C}$ & 35 \\
\hline Temperature viscosity index & $\alpha$ & 0.041 \\
\hline \multicolumn{3}{|r|}{} \\
\hline \multicolumn{2}{|c}{}
\end{tabular}

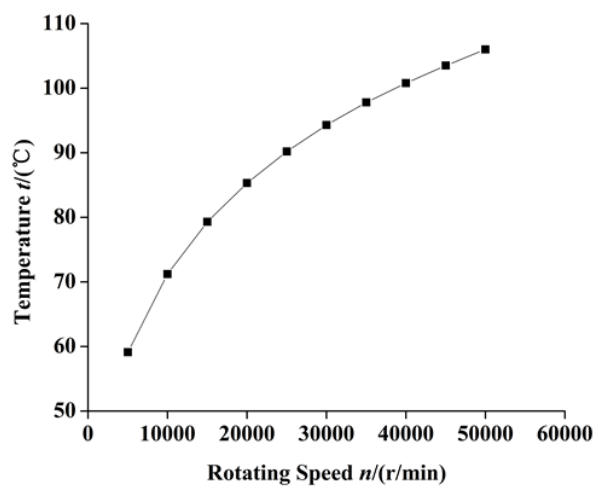

Figure 4. Bearing temperature curve with rotating speed rises.

Bearing stiffness and damping are important parameters affecting the mode of spindle. As shown in
Fig. 5 and 6, under a specify load, the bearing eccentric decreases with the rotating speed increases causes the oil film stiffness varies in different trends in different directions. It also will bring lubricant flow and oil temperature increase. Fig. 7 and 8 shows the bearing eccentric increases with the load increases causes the oil film stiffness increases under a constant rotating speed. It also will bring friction of the bearing and the temperature increase. As shown in Fig. 9 and Fig. 10, if the load and the rotating speed is constant, the stiffness and damping of the bearing have different trends in different directions. Whereas, the absolute values are decrease trend. From these several figures, it is found that the effects of rotational speed, load and temperature on the bearing are mutually restricted.

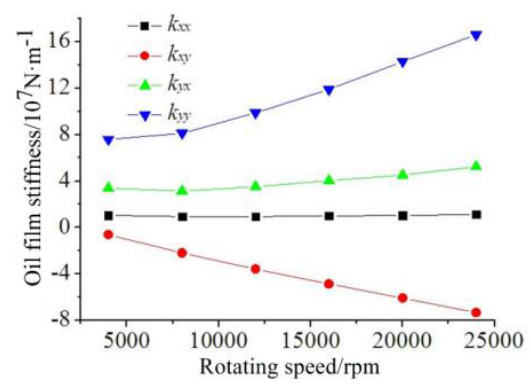

Figure 5. Effect of rotating speed on the stiffness of bearing oil film.

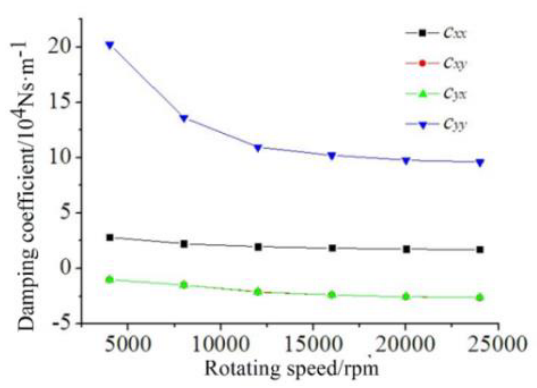

Figure 6. Effect of rotating speed on damping coefficient of bearing.

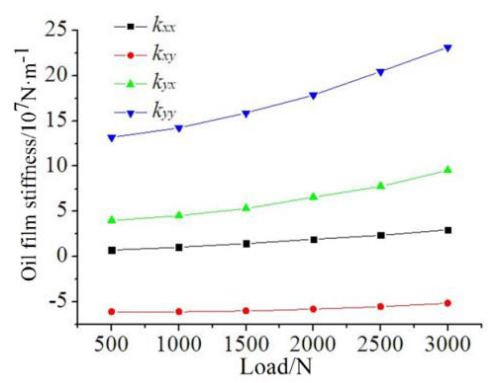

Figure 7. Effect of load on the stiffness of bearing oil film.

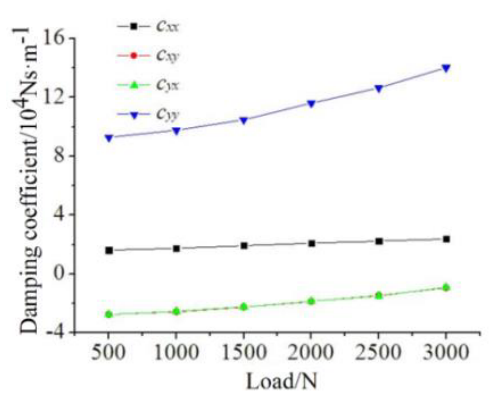

Figure 8. Effect of load on bearing damping coefficient. 


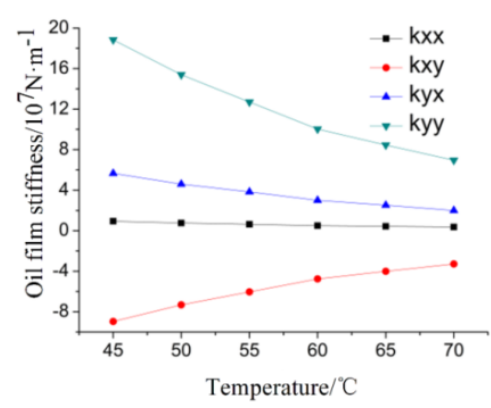

Figure 9. Effect of temperature on bearing stiffness.

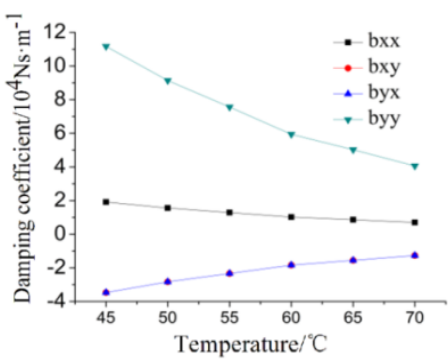

Figure 10. Effect of temperature on damping coefficient of bearing.

Fig. 11 shows the influence of bearing stiffness on the critical speed, all other things being equal. With the increase of the stiffness, the critical speed of each order shows an increasing trend, especially for the higher order. In order to improve the critical speed of the rotor system, if other conditions unchanged, it is necessary to increase the support stiffness of the rotor system. However, to make a substantial increase in the critical speed, bearing stiffness needs to increase exponentially.

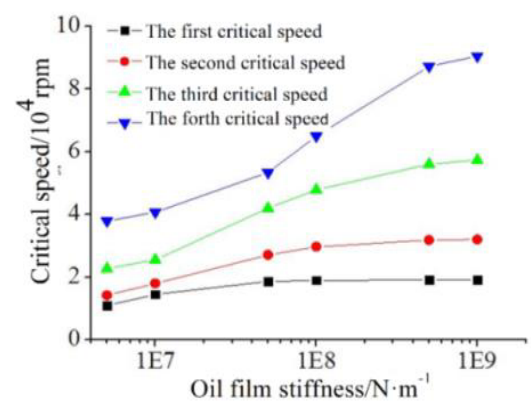

Figure 11. Effect of oil film stiffness on critical speed of rotor system.

As shown in Fig. 12, the damping coefficient of oil film also affects the critical speed. With the increase of the damping coefficient, all other things remaining unchanged, the critical speed is decreased obviously.

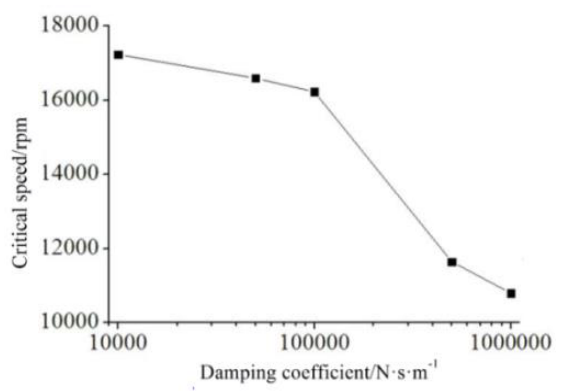

Figure 12. Effect of damping coefficient of bearing on the first order critical speed.
When calculate the rotor dynamic characteristic of the experimental motorized spindle supported by sliding bearings with $100 \mathrm{~N}$ axial load, the influence of temperature and rotational speed on the bearing stiffness and damping coefficient is also considered. Results of the primary four orders of natural frequency and corresponding vibration mode is shown in Fig. 13.

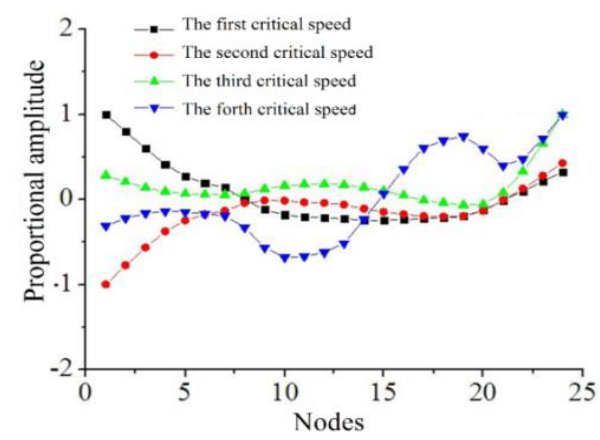

Figure 13. Rotor vibration shape at each order of critical speed.

The critical speed can also be calculated. The results of isotropy sliding bearing rotor system and anisotropy sliding bearing rotor system are shown in Table 2. As can be seen from the table, the calculation results of isotropic bearing model are larger than anisotropic bearing model. It's because of the influence of the cross stiffness and damping coefficient of the oil film are neglected in isotropic bearing model.

Table 2. The rotor system critical speed of the two model

\begin{tabular}{|c|c|c|c|c|}
\hline & $\begin{array}{c}\text { 1st } \\
\text { order }\end{array}$ & $\begin{array}{c}\text { 2nd } \\
\text { order }\end{array}$ & $\begin{array}{c}\text { 3rd } \\
\text { order }\end{array}$ & $\begin{array}{c}\text { 4th } \\
\text { order }\end{array}$ \\
\hline $\begin{array}{c}\text { Isotropic } \\
\text { bearing model } \\
\text { (rpm) }\end{array}$ & 19052 & 30651 & 50876 & 72974 \\
\hline $\begin{array}{c}\text { Anisotropic } \\
\text { bearing model } \\
\text { (rpm) }\end{array}$ & 17774 & 27764 & 49204 & 70538 \\
\hline
\end{tabular}

\subsection{Dynamic experimental verification of the sliding bearing high speed motorized spindle}

Vibration of the spindle front end and motorized spindle shell are measured when the motorized spindle is operating. By comparing the amplitude of vibration under different rotating speed, the critical speed and other parameters can be obtained indirectly. Fig. 14 shows the experimental facilities.

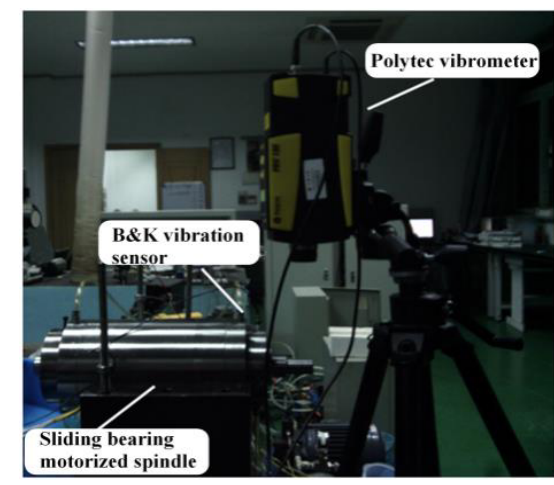

Figure 14. Experimental setup. 
In the experiment, the motorized spindle runs at each speed for 90 minutes till the motorized spindle reaches thermal steady state [19]. Laser Doppler vibration meter Polytec PDV100 is used to measure the vibration value of rotor front end and vibration value of motorized spindle shell is measured by B\&K vibration/noise analyze system.

Fig. 15 shows the vibration amplitude of the spindle at different speeds, Fig. 16 shows the vibration amplitude of the shell at different speeds. As shown in the figures, the amplitude appears to be the first significant maximum at $18000 \mathrm{rpm}$, which is also $300 \mathrm{~Hz}$. This shows that the sliding bearing system reaches the resonance at about $300 \mathrm{~Hz}$, that means the first critical speed of the high speed motorized spindle rotor system supported by the sliding bearing is around $18000 \mathrm{rpm}$. The anisotropy sliding bearing rotor model is more accurate. The isotropy model neglect the affect of cross stiffness and damping coefficient, they can reduce the natural frequency of the rotor system.

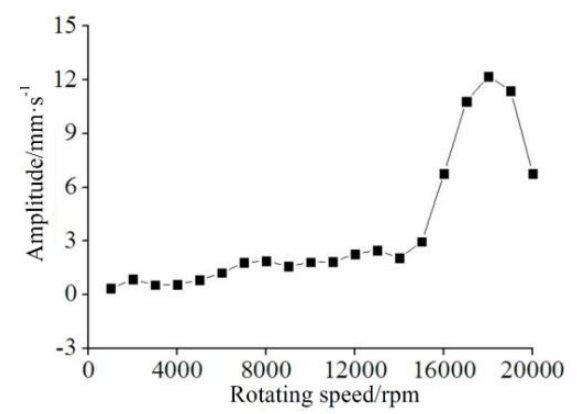

Figure 15. Vibration amplitude of the spindle at each rotating speed.

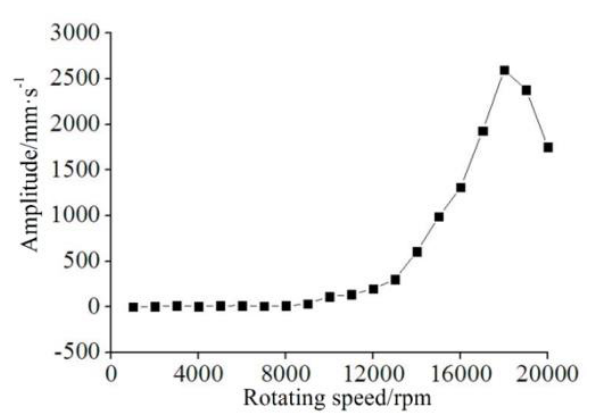

Figure 16. Vibration amplitude of the motorized spindle shell at each rotating speed.

\section{Conclusions}

(1) Due to the extrusion and viscous shearing of the lubricating oil, the sliding bearing will generate large amount of heat when the rotating speed is high, the oil viscosity reduces. The isothermal hydrodynamic lubrication model is no longer applicable. The thermo hydrodynamic lubrication model considering the temperature change and the viscosity temperature effect will be more appropriate in engineering.

(2) Load and rotating speed are important factors to dynamic characteristics of sliding bearing. With the increase of rotating speed, the oil film stiffness varies in different trends in different directions, and the damping coefficient decreases. The film stiffness and damping coefficient are increased with the increase of load.
(3) Experimental study shows the calculated results obtained by the anisotropic sliding bearing rotor model are better than isotropic sliding bearing rotor model. The cross stiffness and damping can reduce the natural frequency of the rotor system.

\section{References}

1. Z.Y. Zhang, Hydrodynamic lubrication theory of sliding bearing. Higher Education Press. Beijing, China, (1986)

2. P. Chang, and B. Lin, Vibration signal analysis of journal bearing supported rotor system by cyclostationarity. Shock \& Vibration, 2014(1), 1-16. (2014)

3. T.H. Machado, and K.L. Cavalca, Modeling of hydrodynamic bearing wear in rotor-bearing systems. Mechanics Research Communications, 69, 15-23, (2015)

4. K.P. Gertzos, P.G. Nikolakopoulos, A.C. Chasalevris, and C.A. Papadopoulos, Wear identification in rotorbearing systems by measurements of dynamic bearing characteristics. Computers \& Structures, 89(12), 55-66 (2011)

5. H. Mccallion, F. Yousif, and T. Lloyd, The analysis of thermal effects in a full journal bearing. Journal of Tribology, 92(4) (1970)

6. W. Xiong, Review on key technology of hydrodynamic and hydrostatic high-frequency motor spindles. Journal of Mechanical Engineering, 45(9), 1-18 (2009)

7. A. Chauhan, R. Sehgal, and R.K. Sharma, Thermohydrodynamic analysis of elliptical journal bearing with different grade oils. Tribology International, 43(11), 1970-1977 (2010)

8. S.S. Li, Y.X. Lu, M.L. Dong, J. Shao, and F. Yu, Modeling and Analysis of High-Speed Journal Bearing Based on Thermal Hydrodynamic Lubrication Theory. Applied Mechanics and Materials 380, 82-86 (November 2013)

9. L. Daquan, T. Zheng, W. Zhang. Thermohydrodynamic study on journal bearings using finite element analysis. China Mechanical Engineering, 17(12), 1285-1289 (2006)

10. J. Durany, J. Pereira, and F. Varas, Dynamical stability of journal-bearing devices through numerical simulation of thermohydrodynamic models. Tribology International, 43(9), 1703-1718 (2010)

11. A.F. Clarens, P.E. Allaire, A. Younan, and S. Wang, Gas-Expanded Lubricants for Increased Energy Efficiency and Related Method and System.U.S. Patent Application No. 13/502,066 (2011)

12. S. Jerebzoff-Quintin, A thermohydrodynamic coupling model of oil aeration turbulent lubrication for journal bearing with interface effect.Journal of Tribology, 137(4), 1718-20 (2015)

13. C. Deepthi and J. Ramathulasi, Parametric Design and Analysis of Two Station Two Spindle Rough Boring and Finish Boring SPM, International Journal of Mechanical Engineering and Robotics Research, 3(4), 612-621, (October 2014) 
14. B.C. Wen, J.C. Gu, S.B. Xia, Advanced rotor dynamics. China Machine Press. Beijing, China. (1999)

15. F. Xie, Calculation of oil film pressure distribution of journal bearing based on finite difference method. Lubrication Engineering (2012).

16. Y. Fu., X. Ma, and J. Zhu, Study on the threedimensional temperature field of circular journal bearing. Mechanical Science \& Technology, 21(5), 711-710 (2002)
17. Y.E. Zhong, Y.Z. He, Z. Wang, The rotor dynamics. Tsinghua University Press. Beijing, China. (1987)

18. L. Yu, Y. Liu, The bearing rotor system dynamics. Xi'an Jiaotong University Press. Xi'an, China. (2001)

19. Q. He, Y. Shen, F. Ren, L. Li, and A. A. Volinsky, Numerical simulation and experimental study of the air-cooled motorized spindle. Proceedings of the Institution of Mechanical Engineers Part C Journal of Mechanical Engineering Science. (2016) 